\title{
AbSence of Bioactivity of Lipid Derivatives of Serotonin
}

\author{
M. Pozdzik ${ }^{1}$, D. Zajac ${ }^{1}$, I. Zasada ${ }^{1}$, Z. Czarnocki², Z. Matysiak ${ }^{3}$, A. Mazzatenta ${ }^{4}$, M. Pokorski ${ }^{1}$ \\ ${ }^{1}$ Department of Respiratory Research, Medical Research Center, Polish Academy of Sciences, Warsaw, Poland; \\ ${ }^{2}$ Department of Organic Chemistry, Faculty of Chemistry, University of Warsaw, Warsaw, Poland; \\ ${ }^{3}$ Institute of Biochemistry and Biophysics, Polish Academy of Sciences, Warsaw, Poland; \\ ${ }^{4}$ Department of Neuroscience and Imaging, 'G. d'Annunzio' University of Chieti-Pescara, Italy
}

\begin{abstract}
Background: Serotonin (5-HT) and its receptors are present in central, the brain stem, and peripheral, the carotid body, tissues controlling the ventilatory responses to hypoxia. The exact action of serotonin and its nature are, however, unsettled. We hypothesized that the discrepant results on the ventilatory action of serotonin could be caused by the inability of serotonin to penetrate into the brain or the plasma membrane lipid bilayers, the target site of signal transduction cascades, after its exogenous administration.

Objective: To study the penetrability of novel lipid derivatives of serotonin of varying fatty acid chain length and number of saturated/unsaturated bonds, the oleic, caprylic, and caprolic amides of 5-HT, into the brain, and their functional effects on the hypoxic ventilatory response in awake rats after systemic administration.
\end{abstract}

Material and methods: Adult Wistar rats were used for the experiments. In the biochemical part of the study, the presence and stability of the compounds tested, after i.p. injection, was assessed in brain extracts using spectrophotometry and thin-layered chromatography. In the functional part, the ventilatory responses to 8 and $12 \%$ hypoxia were compared before and $1 \mathrm{~h}$ after the compound administration using a whole body plethysmography.

Results: The 'lipidized' serotonin compounds turned out to be stable in brain extracts in vitro for up to $3 \mathrm{~h}$ of the test. However, we could not substantiate the presence of any of the compounds in the brain, with either method used, after i.p. administration. Likewise, none of the compounds had any appreciable effect on the profile of the stimulatory hypoxic ventilatory response.

Conclusions: Synthetically attaching lipophilic groups to the serotonin molecule does not make it penetrate into the brain. The lack of serotonin penetrability likely depends on the planarity of its molecule, as it does not seem to depend on the size, number of carbons or bond saturation of the 'lipidized' molecules. Such molecules do not directly interfere with the carotid chemoreceptor-mediated hypoxic ventilatory response. The study failed to substantiate the bioactive potential of the lipid derivatives of serotonin.

\section{INTRODUCTION}

Serotonin (5-hydroxytryptamine, 5-HT) is an amine neurotransmitter in both central and peripheral nervous systems. Synthesized from the amino acid tryptophan by the tryptophan hydroxylase and L-aromatic amino acid decarboxylase, it is metabolized by the omnipresent enzyme monoaminooxydase. The functions of serotonin are very broad. It takes part in regulation of temperature, appetite, mood, sleep cycles, and is the neurotransmitter of major interest in depressive disorders [1, 2]. One of the less publicized effects of serotonin is its role in respiratory regulation. Serotonergic active compounds acutely change eupneic respiratory motor output [3]. Serotonin increases phrenic motoneuron responses through activation of $5-\mathrm{HT}_{2 \mathrm{~A} / \mathrm{C}}$ receptors [4, 5], abundantly present on medullary raphe neurons [6]. 5- $\mathrm{HT}_{2}$ receptors also are key for the long-term hypoxic ventilatory facilitation (LTF); the effect being blocked by an antagonist of these receptors, ketanserin [7,8].

Serotonin also seems essential for the chemical control of respiration, notably for the hypoxic chemoreflex, mediated by central integration of peripheral chemosensory input emanating from the carotid body, a paired organ whose chemoreceptors generate hypoxic hyperventilation [9]. Hypoxia enhances the expression and release of serotonin in the brain stem [10,11] and serotonin has a stimulatory effect on the hypoxic ventilatory response [7]. The stimulatory effect is dampened by ketanserin, which speaks to the involvement of $5-\mathrm{HT}_{2}$ receptors [7], although the two major neuronal motor respiratory outputs, the phrenic and hypoglossal nerves, are not necessarily enhanced in parallel [7]. The action of serotonin in chemical regulation of ventilation is compounded by its presence in the carotid body. Serotonin is expressed in carotid chemoreceptor tissue in humans [12] and rats [13], and the $5-\mathrm{HT}_{2}$ receptors are present in chemoreceptor cells [14]. However, the studies on the role of serotonin in carotid body function have given mixed results. When applied exogenously in the rat, 5-HT augments the afferent nerve activity of the carotid body [15]. Other reports show that the effect of serotonin is fleeting and followed by sustained depression of chemosensory activity [16]. What seems unambiguous 
is that serotonin is engaged in sensory LTF of the carotid body [17] and that any serotonin-related effects are antagonized by ketanserin [14], again pointing to the involvement of $5-\mathrm{HT}_{2}$ receptors. Although serotonin interacts in ventilatory regulation at both central and peripheral levels, the exact determinants of this action, the interplay among serotonin receptor subtypes, and the neurotransmitter's role in respiratory rhythmogenesis are still areas of limited understanding.

Serotonin is not lipid-soluble and as such is believed not to cross the blood-brain barrier (BBB) [18]. The inability to cross biological barriers may be a major handicap in both experimental and therapeutic interventions, directed at either central or systemic supplementation of serotonin or its close derivatives. Moreover, most intricate signaling cascades, in which messenger molecules are generated, are based on the polyunsaturated fatty acid (PUFA) components of neuronal plasma membrane phospholipids [19, 20]; a membrane layer apparently inaccessible for hydrophilic serotonin. These shortcomings might be one reason for often inconclusive, controversial, or differential result in the serotonin experiment. In addition, a plausible deficit of serotonin thought to underlie psychoneurological disorders, e.g., depression, psychoses, and the like, could be more efficiently redressed by serotonin derivatives that would be able to enter the brain after being systemically administered. With these premises in mind, in the present study we examined three de novo synthesized lipidderivatives of serotonin: the oleic, caprylic, and caprolic amides of 5-HT in the context of their penetration into the brain and influence on the respiratory response to hypoxia. The choice of compounds took into account a different number of carbons and unsaturated bonds in the fatty acid chain, the elements that could bear on their bioactive properties $[21,22]$. Overall, the study failed to substantiate the bioactive potential of these novel derivatives of serotonin.

\section{Material And Methods}

The study was approved by a local Ethics Committee for Animal Research. A total of 22 three-four months old male Wistar rats (body weight 280-312 g) were used for the study. All rats were kept with free access to water and rodent chow in an artificial $12 \mathrm{~h}$ light-dark cycle, with the lights on at 8 a.m., temperature of $21 \pm 2{ }^{\circ} \mathrm{C}$, and humidity of $50-60 \%$. The experiments consisted of two parts. In the first part (9 rats) we investigated whether the novel lipid-soluble derivatives of serotonin penetrate into the rat brain after in vivo intraperitoneal injection. The aim of the second part (13 rats) was to establish whether these compounds influence the respiratory response to hypoxia in conscious rats.

\section{Lipid Derivatives of Serotonin}

Three lipid derivatives of serotonin were subject of the study: N-oleoyl-serotonin (C18-5HT), N-caprylserotonin (C8-5HT) and N-caproyl-serotonin (C6$5 \mathrm{HT})$. All of them were synthesized de novo at the Faculty of Chemistry of the University of Warsaw and were generously provided for this study. Purity of the compounds was confirmed using thin-layered chromatography (TLC) which was carried out on precoated HPTLC silica gel $60 \mathrm{~F}_{245}$ plates (Merck KGaA, Darmstadt, Germany) with chloroform/methanol (95:5 v/v) as solvents. Table 1 presents structure details of the compounds studied. Graphic representations of the chemical structures of the compounds tested are shown in Fig. 1.
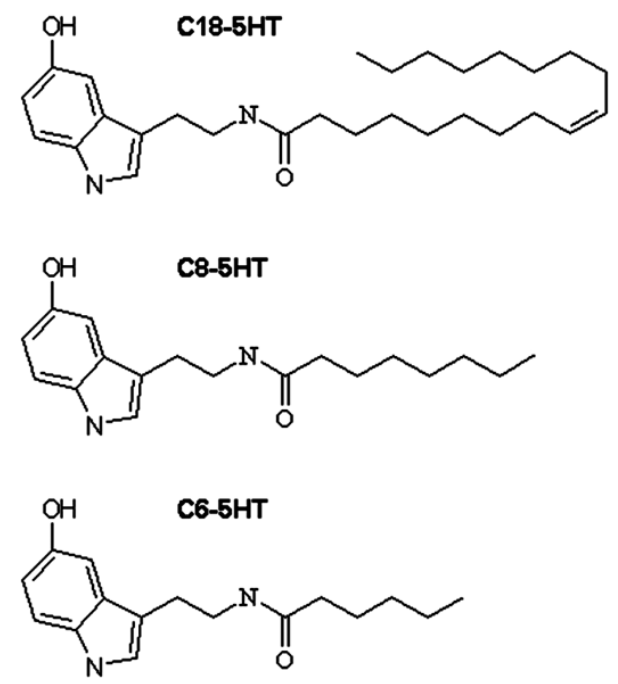

Fig. 1. Chemical structures of 'lipidized' serotonin derivatives: of N-oleoyl-serotonin (C18-5HT), N-capryl-serotonin (C8-5HT), and N-caproyl-serotonin (C6-5HT).

\section{COMPOUNDS STABILITY IN VITRO}

Before performing biological experiments, we had checked the stability of the three derivatives in vitro during 3-h incubation in Krebs buffer and in a crude brain membranes solution. Briefly, Krebs solution containing (in mmol/l) NaCl 118.4, $\mathrm{KCl} 6, \mathrm{NaH}_{2} \mathrm{PO}_{4}$ x $2 \mathrm{H}_{2} \mathrm{O} 0.6, \mathrm{MgSO}_{4} 1.2$, and $\mathrm{NaHCO}_{3} 25$ was prepared and equilibrated to $\mathrm{pH}$ of 7.4 with $\mathrm{CO}_{2}$. Crude brain membranes were prepared as described by Xu et al [23]. The rat was anesthetized with $\alpha$-chloralose and urethane (35 and $800 \mathrm{mg} / \mathrm{kg}$, ip, respectively) and sacrificed by perfusion with ice-cold $0.9 \% \mathrm{NaCl}$ through the heart. The brain was rapidly enucleated from the

Table 1. Lipid derivatives of serotonin studied.

\begin{tabular}{lcccc}
\hline \multicolumn{1}{c}{ Name of derivative } & Molecular formula & $\begin{array}{c}\text { Number of carbons } \\
\text { in the lipid chain }\end{array}$ & $\begin{array}{c}\text { Number of unsaturated } \\
\text { bonds }\end{array}$ & $\begin{array}{c}\text { Molecular mass } \\
(\mathrm{g} / \mathrm{mol}, \mathrm{Da})\end{array}$ \\
\hline N-oleoyl-serotonin (C18-5HT) & $\mathrm{C}_{28} \mathrm{H}_{44} \mathrm{~N}_{2} \mathrm{O}_{2}$ & 18 & 1 & 440.67 \\
N-capryl-serotonin (C8-5HT) & $\mathrm{C}_{18} \mathrm{H}_{26} \mathrm{~N}_{2} \mathrm{O}_{2}$ & 8 & 0 & 302.42 \\
N-caproyl-serotonin (C6-5HT) & $\mathrm{C}_{16} \mathrm{H}_{22} \mathrm{~N}_{2} \mathrm{O}_{2}$ & 6 & 0 & 274.37 \\
\hline
\end{tabular}


skull $(\mathrm{m}=1.84 \mathrm{~g})$ and homogenized in $5 \mathrm{v} / \mathrm{m}$ of the isolation buffer containing (in mmol/l) $\mathrm{MgCl}_{2} \times 6$ $\mathrm{H}_{2} \mathrm{O} 2.5, \mathrm{KHCO}_{3}$, Tris x $\mathrm{HCl} 2$, EDTA 3, benzamidine 0.1, and PMSF/EtOH 0.1. Homogenate was centrifuged at $2000 \mathrm{x} g, 4^{\circ} \mathrm{C} 10 \mathrm{~min}$. The supernatant was taken as crude brain membranes. Calcium chloride was added to both solutions at a final concentration of 2.5 $\mathrm{mmol} / 1$. Then, C18-5HT, C8-5HT, and C6-5HT were added to $5 \mathrm{ml}$ of the solution to reach a working concentration of $1 \mathrm{mmol} / 1$. In parallel, controls containing pure Krebs and membrane solutions were prepared. After 1, 30, 60, 90, 120, and $180 \mathrm{~min}$, half milliliter samples were taken from the Krebs, membranes, and control solutions containing all compounds. Then, $50 \mu \mathrm{l}$ of $8 \%$ trichloroacetic acid were added to pellet proteins and the whole was centrifuged at $2000 \mathrm{x} g$ for $4 \mathrm{~min}$ at $4^{\circ} \mathrm{C}$. The supernatants were extracted 4 times with $0.25 \mathrm{ml}$ chloroform, the organic phases collected, dried under nitrogen and analyzed with a Cintra $10 \mathrm{e}$ UV/VIS Spectrophotometer equipped with Spectral 1.70 software (GBC Scientific Equipment Pty Ltd., Victoria, Australia).

\section{Compounds Penetration into the Brain after SYSTEMIC ADMINISTRATION}

Eight male Wistar rats were divided into four groups of two rats each. Each group received a different derivative: $\mathrm{C} 18-5 \mathrm{HT}$ - $50 \mathrm{mg} / \mathrm{kg}, \mathrm{C} 8-5 \mathrm{HT}-30 \mathrm{mg} / \mathrm{kg}$, and C6-5HT - $27 \mathrm{mg} / \mathrm{kg}$, all dissolved in $0.3 \mathrm{ml}$ of DMSO, and $0.3 \mathrm{ml}$ of DMSO alone as a control, all given i.p. The dose of each compound was equal to $0.1 \mathrm{~mol} / \mathrm{kg}$ of body weight, which corresponds to the concentration of serotonin suggested by Hilaire et al [24] as being bioactive in animal models for respiratory studies.

An hour after injection of a tested substance, the animals were anesthetized and sacrificed as outlined above. The brain was enucleated, weighed, homogenized in $20 \mathrm{v} / \mathrm{m}$ of chloroform:methanol $2: 1 \mathrm{v} / \mathrm{v}$ and left overnight at $4{ }^{\circ} \mathrm{C}$. Then, the mixture was dried under nitrogen to a volume of $3 \mathrm{ml}$ and the lipids were extracted 3 times with chloroform and water $(1: 1 \mathrm{v} / \mathrm{v}$, $4 \mathrm{ml}$ ) to remove hydrophilic substances. An organic, lipid-containing fraction was dried under nitrogen and stored at $-80^{\circ} \mathrm{C}$ until analysis.

The analysis consisted of two steps. First, the sample was dissolved in $2 \mathrm{ml}$ of chloroform. From that volume, $0.3 \mathrm{ml}$ was taken for UV/VIS spectral analysis. In parallel, spectra of the control (standard) solutions of each substance at a concentration of $1 \mathrm{mg} / \mathrm{ml}$ were performed. The second step consisted of TLC of the samples. Twenty microliter of pure lipid and the investigated membrane solution were applied, alongside the $20 \mu \mathrm{l}$ of the standard, on silica gel $60 \mathrm{~F}_{245}$ plates (Merck KGaA, Darmstadt, Germany) with chloroform:methanol $(95: 5, \mathrm{v} / \mathrm{v})$ as solvents. Then, all plates were visualized by iodine vapors and immediately photographed.

Concerning the spectral analysis, we compared the characteristic points (maximum and minimum) and shape of the spectra among the samples, control lipids, and standards. In chromatographic studies, retention factors $\left(\mathrm{R}_{\mathrm{f}}\right)$, calculated as the ratio between the migration distance of a substance to that of the solvent front, were taken under consideration.

\section{Functional Studies - The Hypoxic Ventilatory RESPONSE}

Thirteen awake rats were used for the functional ventilatory studies. Seven rats were used for the tests with $\mathrm{N}$-oleoyl-serotonin (C18-5HT), and 3 each for $\mathrm{N}$ capryl-serotonin (C8-5HT) and N-caproyl-serotonin (C6-5HT). Ventilatory measurements were performed in a whole body single-chamber plethysmograph (model PLY3223, Buxco Electronics, Wilmington, NC). Pressure difference between the experimental and reference chambers was measured with a differential pressure transducer. The pressure signal was amplified and integrated with data analysis software (Biosystem XA for Windows SFT3410 v. 2.9, Buxco Electronics, Wilmington, $\mathrm{NC})$. Tidal volume $\left(\mathrm{V}_{\mathrm{T}}\right)$, respiratory frequency

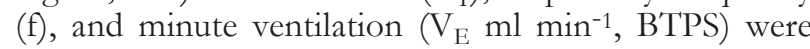
computed breath-by-breath and analyzed off-line. Ten second averages were taken before the half-minute time points during the course of hypoxic exposure.

The experiment started with a $10 \mathrm{~min}$ period of the acclimation of a rat to the chamber, breathing ambient air. Then, the animal was randomly exposed to two decreasing levels inspired oxygen: 12 and 8\% $\mathrm{O}_{2}$ balanced with $\mathrm{N}_{2}$ in the poikilocapnic setting. Hypoxic tests took $3 \mathrm{~min}$, followed by recovery in room air. Hypoxic exposures were separated by a $15 \mathrm{~min}$ recovery interval in air. Next, the animal was injected with a compound tested, in the doses outlined above, and the tests were repeated after the time elapse of $60 \mathrm{~min}$. In the protocol employed for the study, the hypoxic response in a pharmacologically treated animal was compared with the response in the same untreated animal. Therefore, each animal served as its own control.

A set of experiments with each compound tested was treated as a separate entity for statistical elaboration. All ventilatory data were normalized for weight in $\mathrm{kg}$ and were expressed as means $\pm \mathrm{SE}$. Minute ventilation VE was the variable of the major meaning, and the tidal and frequency components were analyzed only if significant changes were noted in $\mathrm{V}_{\mathrm{E}}$. The course of each hypoxic response was represented by the group mean values at sequential $30 \mathrm{~s}$ time points. Statistical comparison concerned the three main time points of interest in $\mathrm{V}_{\mathrm{E}}$ : basal, peak at $30 \mathrm{~s}$, and depressant nadir at test-end. The Friedmann test was used to compare these three time points in each response, followed, if significant, by a Wilcoxon test for comparisons among these three time points. Pair-wise comparison of differences at the corresponding time points between control and compound's effects was performed with a Wilcoxon test. $\mathrm{P}<0.05$ was assumed to indicate statistical significance of differences.

\section{RESULTS}

\section{STABILITY IN VITRO}

All three tested derivatives of serotonin turned out to be fairly stable during the $3 \mathrm{~h}$ observation time. Stability of N-oleoyl-serotonin (C18-5HT) in Krebs solution and in crude brain membranes is demonstrated in Fig. 

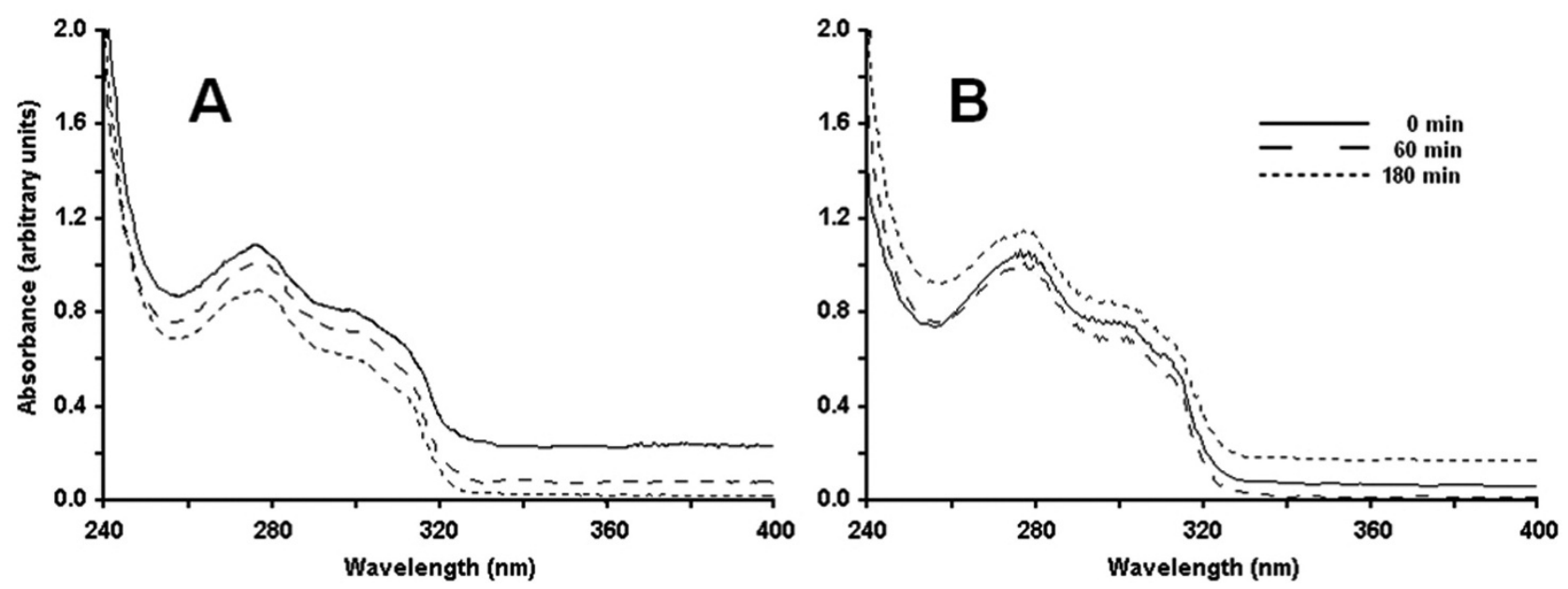

Fig. 2. Spectrophotometric spectra showing stability of $\mathrm{C} 18-5 \mathrm{HT}$, after its incubation at $36^{\circ} \mathrm{C}$ at the time periods indicated in the legend, in Krebs (Panel A) and in crude brain membranes solutions (Panel B).

$3 \mathrm{~A}$ and B. In both solutions, C18-5HT had a clear and repetitive spectral shape during the entire experiment. The maximum was located at $275 \mathrm{~nm}$ and minimum at $259 \mathrm{~nm}$, with a secondary peak at $310 \mathrm{~nm}$. The spectra remained unchanged over $3 \mathrm{~h}$.

C8-5HT and C6-5HT also showed an unchanged pattern of shape over $3 \mathrm{~h}$ in both solutions (graphical data not shown). The former's maximum was at 276 $\mathrm{nm}$ and minimum at 258-260 nm. The latter's maximum was at 277 and minimum at $258 \mathrm{~nm}$, with both compounds having a secondary peak at $310 \mathrm{~nm}$. Of note, the shapes of each serotonin derivative's spectra were somehow different in biological samples than those in the control solutions, even though the maxima and minima were at the same wave length.

\section{Penetration into the Brain}

Here, each compound was injected i.p. and the extract of brain membranes was investigated after $1 \mathrm{~h}$ and the spectra compared with standards. Fig. 3. exemplifies the results obtained with $\mathrm{C} 18-5 \mathrm{HT}$. The spectrum for the lipids obtained from the control untreated rats,

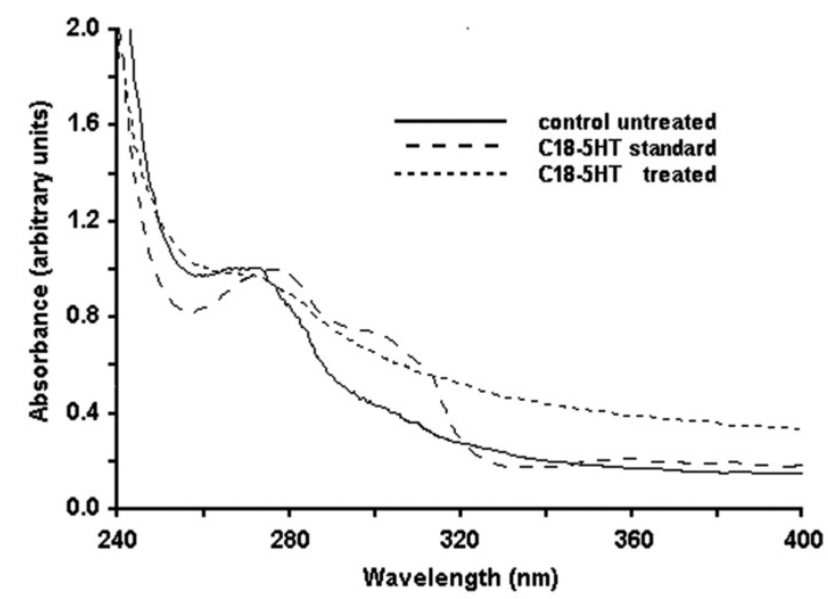

Fig. 3. Spectra of brain extracts obtained from the control untreated (lipids standard), C18-5HT standard, and i.p. C185 HT treated rats. taken as lipids standard, showed a maximum at 273 $\mathrm{nm}$ and minimum at $261 \mathrm{~nm}$. The C18-5HT standard had two maxima: at 275 and $310 \mathrm{~nm}$, and a minimum at $259 \mathrm{~nm}$. The spectrum obtained from the brain extracts of C18-5HT treated rats was different. We failed to detect local maxima and minima within the range of $240-400 \mathrm{~nm}$, where these characteristic points were detected for standards. Additionally, a subtraction of the lipids spectrum from the C18-5HT spectra did not result in clearly shaped standard-like spectra. The shape of brain extracts spectra obtained from the rats injected with the other two derivatives of serotonin did not bear any resemblance to the standards either (data not shown).

In TLC experiments, $\mathrm{R}_{\mathrm{f}}$ for the $\mathrm{N}$-oleoyl-serotonin (C18-5HT) standard was 0.44. No spots appeared at this level either concerning the samples prepared from untreated or C18-treated rats (Fig. 4). $\mathrm{R}_{\mathrm{f}}$ for N-capryl-

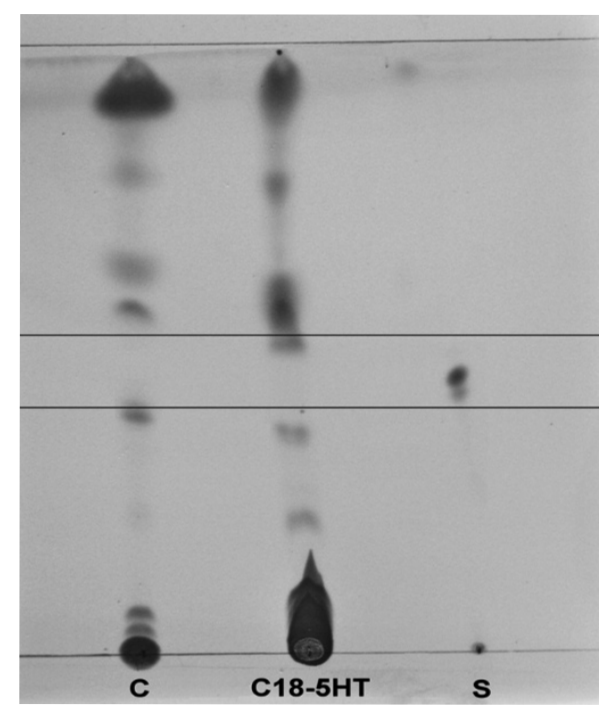

Fig. 4. TLC plate showing lipid extracts prepared from brains of control untreated $(\mathrm{C})$, taken as lipids standard, and $\mathrm{N}$ oleoyl-serotonin (C18-5HT)-treated (1 h after $50 \mathrm{mg} / \mathrm{kg}$, i.p.) rats, alongside the C18-5HT standard (S). There were no lipid spots at the level of the standard shown by a rectangular. See Material and Methods for analytical details. 


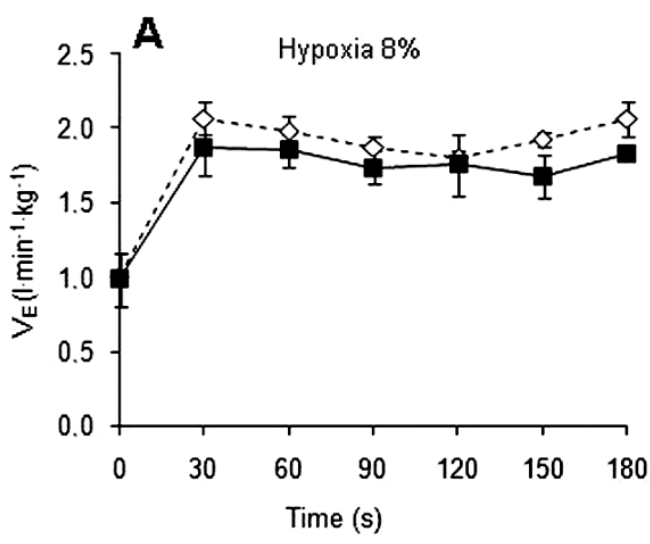

Fig. 5. Course of minute ventilation responses to 8 and $12 \%$ hypoxia before (control) and after $\mathrm{N}$-oleoyl-serotonin (C18-5HT; $50 \mathrm{mg} / \mathrm{kg}$ ) i.p. administration in awake rats. serotonin (C8-5HT) were 0.26 and 0.32 and for $\mathrm{N}$ caproyl-serotonin (C6-5HT) were 0.21 and 0.26 . In neither case were there spots detected at the same levels in preparations from C8-5HT and C6-5HT injected rats (data not shown).

\section{Hypoxic Ventilatory Response}

The profile of the hypoxic ventilatory responses, assessed from its minute volume $\left(\mathrm{V}_{\mathrm{E}}\right)$, in the control condition and after administration of C18-5HT in a dose of $50 \mathrm{mg} / \mathrm{kg}$, i.p., is demonstrated in Fig. 5. The response consisted of early stimulation of ventilation, which achieved its peak at $30 \mathrm{~s}$ of hypoxia, followed by a slow decline. The decline, corresponding to the ventilatory hypoxic depression, did not result in a significant difference in $\mathrm{V}_{\mathrm{E}}$ compared with its peak, likely due to a shortness of the hypoxic test. In the control, $\mathrm{V}_{\mathrm{E}}$ increased from the baseline $1.01 \pm 0.01$ to 2.07 $\pm 0.12 \mathrm{l} / \mathrm{min} / \mathrm{kg}$ for $8 \%$ hypoxia and from $1.19 \pm 0.14$ to $1.62 \pm 0.03 \mathrm{l} / \mathrm{min} / \mathrm{kg}$ for $12 \%$ hypoxia $(\mathrm{P}<0.05)$. The stimulatory responses were achieved by increases in both frequency and tidal components of ventilation. After C18-5HT, the profile and magnitude of the hypoxic $\mathrm{V}_{\mathrm{E}}$ responses did not change appreciably (Fig. 5). Likewise, the other two derivatives of serotonin tested, C8-5HT and C6-5HT, failed to appreciably influence the responses to hypoxia (data not shown).

\section{Discussion}

Given the multifactorial nature of neurotransmitterrelated respiratory regulation, researchers theorized that serotonin was the neurotransmitter of interest. Therefore, there have been numerous attempts to identify reproducible alterations in respiration in response to application of serotonin or agents interfering with its receptors. Although serotonin is elaborated in hypoxia, its role in the hypoxic ventilatory response is debatable and the studies on the issue are hampered by the inability of serotonin to cross the blood brain barrier (BBB). Insolubility of serotonin in lipids limits its usefulness as a potential neurotherapeutic and confounds the experimental studies.

For a molecule to diffuse through the BBB, it should have a sufficient amount of lipid solubility. With that in mind, in the present study we set out to determine the passage through the BBB of three novel synthetic 'lipidized' derivatives of serotonin. Fur- thermore, intracellular signal transduction pathways are based on the polyunsaturated fatty acids (PUFAs) components of the neuronal plasma membrane phospholipids [19]. Such components, when membranebound, modulate membrane fluidity and functional properties of membrane proteins [20]. Therefore, we reasoned that diffusion of 'lipidized' molecules of serotonin would be the brain-targeting way to study the effects of exogenous serotonin. We also wished to examine the respiratory-related effects of these compounds, as serotonin and its $5-\mathrm{HT}_{2}$ receptors are clearly involved in the chemical control of respiration at both central and peripheral levels [7, 11] and PUFAs are involved in signal transduction mechanisms of chemosensory neurons [25]. The plausible effects on the hypoxic ventilatory response of the 'lipidized' derivatives of serotonin could serve as an index of their bioactivity.

The findings of the study were clearly negative on both counts. Even though the serotonin molecule turned out to be synthetically open to lipophilic modifications, as it was successfully bonded at the N-terminal with different lipophilic groups, its diffusion through the brain capillaries remained impeded. None of the compounds were recovered from brain tissue after systemic injection and none had any countable effect on hypoxic ventilation.

That the lipid-derivatives of serotonin investigated in the present study do not penetrate into the brain was a rather unexpected finding which ran against our working hypothesis, and which is not easily explicable. Attaching lipophilic groups to a compound is a way to make them penetrate the BBB. For instance, N-oleoyldopamine, a condensation product of oleic acid and dopamine of the molecular mass similar to that of C18-5HT used in the present study, 418 and $441 \mathrm{Da}$, respectively, does cross the barrier [26]. Molecular mass of both compounds is well below the limit of about $700 \mathrm{Da}$ proposed by Scherrman et al [27] for lipophilic substance penetrability into the brain. Therefore, it is unlikely that the lack of penetrability of C18-5HT could have to do with the free fatty acid chain, or with its length, in terms of the number of carbons, or the number of saturated/unsaturated bonds, all potential modifiers of penetration through membranes [21, 22]; as we purposefully used compounds with varying characteristics to this end. In all likelihood, the issue lies with the structure of the serotonin ring which is chemically 'planar' and, irrespective 
of the lipophilic modifiers, gets locked into the barrier rather than crossing it, particularly that it becomes bulky after attaching a fatty acid chain at the N-terminus. Lack of penetration into the brain of the other two compounds, caproic (C6-5HT) and caprylic (C8$5 \mathrm{HT})$, whose fatty acid moieties are saturated and relatively short, is not readily explicable. These molecules of 274 and $302 \mathrm{Da}$, respectively, are amphiphilic. They are too big to penetrate the membranes as hydrophilic substances, particularly that the carbon chains of lipids cause them to be immiscible with the aqueous phase, and their lipophilicity is likely too small to allow them to penetrate as lipophilic compounds.

One another reason for the lack of penetrability of the serotonin-derived compounds employed in the present study could be that, as opposed to the amide of dopamine mentioned above which is native to the brain [28], these compounds are purely synthetic and therefore foreign to brain tissue. Alternative study designs would be required to resolve this issue. Since we found that the lipid derivatives of serotonin did not cross the BBB, any central serotonin-like biological effects on respiration could hardly be expectable.

In the present study we found the lipid derivatives of serotonin are fairly stable in brain tissue extracts for up to 3 hours. The finding is in accord with a longterm integrity of the only other fatty acid amide of serotonin reported in the literature, arachidonoylserotonin (AA-5HT). AA-5HT inhibits fatty acid amide hydroxylase (FAAH) and blocks the vanilloid TRPV1 receptors in a neuroblastoma cell line in vitro $[29,30]$. That suggests that other amides of serotonin also may be resistant to decomposition by FAAH. The penetrability into the brain of AA-5HT has not been studied.

In the present study we did not directly measure the penetrability of the serotonin derivatives into the carotid body (CB), a sensory organ which generates the hypoxic chemoreflex. The $\mathrm{CB}$ is not equipped with a barrier of the BBB type and is a target tissue for $\mathrm{N}$-oleoyl-dopamine [31]; the compound also affecting ventilatory responses to hypoxia [32]. It is a reasonable assumption that lipid-derivatives of serotonin also could be taken up by the CB. Functionally, however, administration of serotonin derivatives failed to cause appreciable changes in the hypoxic ventilatory response. Serotonin and $5-\mathrm{HT}_{2}$ receptors are present in carotid chemoreceptors. Several studies have attempted to examine the effects of serotonin or its receptors antagonists on hypoxia-induced chemoreceptor excitation. In a recent comprehensive study performed on ex vivo carotid bodies to avoid secondary, blood pressure-related effects on carotid chemosensory discharge, Jacono et al [33] have found that 5-HT has no effect on the magnitude or onset of the hypoxic sensory response. Our present findings are, generally, in line with that study, showing no effect of lipid derivatives of serotonin on the hypoxic ventilatory response in vivo in conscious rats. Others have reported on the elaboration of brain serotonin in hypoxia $[10,11]$ and hypoxic ventilatory stimulation by serotonin [7]. Thus, the issue remains contentious and may be species dependent.
We conclude that lipid-soluble derivatives of serotonin do not hold promise in solving the uncertainties concerning the role of this neurotransmitter in respiratory regulation. The vastly complex neural respiratory system and the serotonin molecule are constructed in such a manner as to not take advantage of the natural diffusion processes for more general use of serotonin. The study failed to substantiate the bioactive potential of lipid derivatives of serotonin. Other chemical delivery systems should be designed to shepherd serotonin into the brain.

Conflicts of interest: No conflicts of interests were declared by the authors in relation to this article.

\section{REFERENCES}

1. Mohamed-Zadeh L, Moses L, Gwaltney-Brant S. Serotonin: A review. J Vet Pharmacol Therap 2008; 31: 187-99.

2. Green A. Neuropharmacology of 5-hydroxytyramine. Br J Pharmacol 2006; 147: S145-52.

3. Richmonds CR, Hudgel DW. Hypoglossal and phrenic motoneuron responses to serotonergic active agents in rats. Respir Physiol 1996; 106: 153-60.

4. Lindsay AD, Feldman JL. Modulation of respiratory activity of neonatal rat phrenic motoneurones by serotonin. J Physiol (Lond) 1993; 461: 213-33.

5. Morin D, Monteau R, Hilaire G. Compared effects of serotonin on cervical and hypoglossal inspiratory activities: an in vitro study in the newborn rat. J Physiol (Lond) 1992; 451: 605-29.

6. Jacobs BL, Azmitia EC. Structure and function of the brain serotonin system. Physiol Rev 75: 165-229, 1992.

7. Kinkead R, Mitchell GS. Time-dependent hypoxic ventilatory responses in rats: effects of ketanserin and 5-carboxamidotryptamine. Am J Physiol Regul Integr Comp Physiol 1999; 277: R658-66.

8. Bach KB, Mitchell GS. Hypoxia-induced long-term facilitation of respiratory activity is serotonin dependent. Respir Physiol 1996; 104: 251-60.

9. Pokorski M. Control of Breathing. In: Rehabilitation of the Patient with Respiratory Disease. NS Cherniack, MD Altose, I Homma (eds.), McGraw-Hill: New York, 1999; pp. 69-86.

10. Erickson JT, Millhorn DE. Hypoxia and electrical stimulation of the carotid sinus nerve induce Fos-like immonoreactivity within catecholaminergic and serotoninergic neurons of the rat brainstem. J Comp Neurol 1994; 348: 161-82.

11. Teppema LJ, Veening JG, Kranenburg A, Dahan A, Berkenbosh A, Olievier C. Expression of c-fos in the rat brainstem after exposure to hypoxia and to normoxic and hyperoxic hypercapnia. J Comp Neurol 1997; 388: 169-90.

12. Habeck JO, Pallot DJ, Kummer W. Serotonin immunoreactivity in the carotid body of adult humans. Histol Histopathol 1994; 9: 227-32.

13. Grönblad M, Liesi P, Rechardt L. Serotonin-like immunoreactivity in rat carotid body. Brain Res 1983; 276: 348-50.

14. Zhang M, Nurse CA. Does endogenous 5-HT mediate spontaneous rhythmic activity in chemoreceptor clusters of rat carotid body? Brain Res 2000; 872: 199-203.

15. Sapru HN, Krieger AJ. Effect of 5-hydroxytryptamine on the peripheral chemoreceptors in the rat. Res Commun Chem Pathol Pharmacol 1977; 16: 245-50.

16. Nishi K. The action of 5-hydroxytryptamine on chemoreceptor discharges of the cat's carotid body. Br J Pharmacol 1975; 55: 27-40. 
17. Peng YJ, Yuan G, Jacono FJ, Kumar GK, Prabhakar NR. 5-HT evokes sensory long-term facilitation of rodent carotid body via activation of NADPH oxidase. J Physiol 2006; 576: 289-95.

18. Carley D, Radulovacki M. Role of peripheral serotonin in the regulation of central sleep apneas in rats. Chest 1999; 115: 1397-401.

19. Piomelli D, Greengard P. Lipoxygenase metabolites of arachidonic acid in neuronal signalling. Trends Pharmacol Sci 1990; 11: 367-73

20. Stubbs CD, Smith AD. The modification of mammalian membrane polyunsaturated fatty acid composition in relation to membrane fluidity and function. Biochim Biophys Acta 1984; 779: 89-137.

21. de Boer A, van der Sandt I, Gaillard P. The role of drug transporters at the blood brain barrier. Annul Rev Pharmacol Toxicol 2003; 43: 629-56.

22. Habgood MD, Begley DJ, Abbot N. Determinants of passive drug entry into the central nervous system. Cell Mol Neurobiol 2000; 20: 231-53.

23. Xu X, de Pergola G, Eriksson PS, Fu L, Carlsson B, Yang S, Eden S, Bjorntorp P. Postreceptor events involved in the upregulation of beta-adrenergic receptor mediated lipolysis by testosterone in rat white adipocytes. Endocrynology 1993; 132: 1651-7.

24. Hilaire G, Morin D, Lajard A-M and Minteau R. Changes in serotonin metabolism may elicit obstructive apnoea in the newborn rat. J Physiol 1993; 466: 367-82.

25. Mazzatenta A, Di Giulio C, Pokorski M. Interaction of arachidonic acid with electrogenic properties of mouse chemosensory neurons. Eur J Med Res 2010; (in this volume).

26. Zajac D, Matysiak Z, Czarnocki Z, Pokorski M. Membrane association of $\mathrm{N}$-oleoyl-dopamine in rat brain. J Physiol Pharmacol 2006; 57 Suppl 4: 403-8.

27. Scherrmann J-M. Drug delivery to brain via the bloodbrain barrier. Vasc Pharmacol 2002; 38: 349-54.

28. Chu C, Huang S, De Petrocellis L, Bisogno T, Ewing SA, Miller JD, Zipkin RE, Daddario N, Appendino G, Di Marzo V, Walker JM. N-oleoyldopamine, a novel endogenous capsaicin-like lipid that produces hyperalgesia. J Biol Chem 2003; 278: 13633-9.
29. Bisogno T, Melck, D, De Petrocellis L, Bobrov MYu Gretskaya NM, Bezuglov VV, Sitachitta N, Gerwick WH, Di Marzo V. Arachidonoylserotonin and other novel inhibitors of fatty acid amide hydrolase. Biochem Biophys Res Commun 1998; 248: 515-22.

30. Maione S, De Petrocellis L, de Novellis V, Schiano Moriello A, Petrosino S, Palazzo E, Sca Rossi F, Woodward DF, Di Marzo V. Analgesic actions of N-arachidonoyl-serotonin, a fatty acid amide hydrolase inhibitor with antagonistic activity at vanilloid TRPV1 receptors. Br J Pharmacol 2007; 150: 766-81.

31. Pokorski M, Zajac D, Kapuscinski A, Matysiak Z Czarnocki Z. Accumulation of radiolabeled N-oleoyldopamine in the rat carotid body. Adv Exp Med Biol 2006; 580: 173-8.

32. Zajac D, Pokorski M. N-oleoyl-dopamine and carotid body chemosensory responses in the rat. $86^{\text {th }}$ Annual Meeting of the German Physiological Society, March 2528, 2007, Hannover, Germany; Acta Physiologica 2007; 189 Suppl 653: O17-6, p.66 (Abstract).

33. Jacono FJ, Peng Y-J, Kumar GK, Prabhakar NR. Modulation of the hypoxic sensory response of the carotid body by 5 -hydroxytryptamine: Role of the $5-\mathrm{HT}_{2}$ receptor. Respir Physiol Neurobiol 2005; 145: 135-42.

Address for correspondence:

M. Pokorski

Medical Research Center

Polish Academy of Sciences

5, Pawinskiego St.

02-106, Warsaw

Poland

Phone.fax: +48 226086622

E-mail: mpokorski@cmdik.pan.pl 